\title{
Fatores relacionados à mortalidade infantil por anomalias congênitas, Paraná, Brasil
}

\section{0-2019}

\author{
Factors related to infant mortality for congenital anomalies, Paraná, Brazil 2010-2019 \\ Factores relacionados con la mortalidad infantil por anomalías congénitas, Paraná, Brasil 2010-
}

\section{Resumo}

Objetivo: investigar os fatores relacionados aos óbitos infantis por anomalias congênitas nos municípios pertencentes à $14^{\text {a }}$ Regional de Saúde do estado do Paraná, no período de 2010 a 2019. Metodologia: estudo transversal baseado em dados secundários do Sistema de Informação dos Nascidos Vivos, Sistema de Informação sobre Mortalidade e do Sistema de Informação de Agravos de Notificação. Resultados: a detecção das anomalias antes da alta hospitalar evidenciou-se como fator de proteção para o óbito. Já, os seguintes fatores foram associados ao óbito: crianças com baixo peso ao nascer $(\mathrm{OR}=5,7 ; \mathrm{IC} 95 \%=3,37-9,77 ; \mathrm{p}<0,001)$ e filhos de mães sem companheiro $(\mathrm{OR}=1,8 ; \mathrm{IC} 95 \%=$ 1,10-2,98; $\mathrm{p}=0,019)$, número inadequado de consultas de pré-natal $(\mathrm{OR}=1,7 ; \mathrm{IC} 95 \%=1,01-2,84 ; \mathrm{p}=0,047)$, tipo de gravidez dupla e mais $(\mathrm{OR}=9,3 ; \mathrm{IC} 95 \%=1,63-53,52 ; \mathrm{p}=0,031)$, parto prematuro $(\mathrm{OR}=4,0 ; \mathrm{IC} 95 \%=2,39-6,85$; $\mathrm{p}<0,001)$, e Apgar inadequado no primeiro $(\mathrm{OR}=5,5$; IC95\%=3,28-9,32; $\mathrm{p}<0,001)$ e quinto minuto de vida $(\mathrm{OR}=8,9$; IC95\%= 4,46-17,59; p<0,001). Conclusão: vários fatores estão associados com a mortalidade por anomalias congênitas. Portanto, ressalta-se a importância da prevenção desses fatores por meio do fortalecimento do processo de vigilância epidemiológica das anomalias, planejamento familiar, pré-natal adequado e diagnóstico precoce para melhora da qualidade de vida e sobrevida de crianças com anomalias.

Palavras-chave: Anormalidades congênitas; Fatores de risco; Mortalidade infantil; Saúde pública.

\begin{abstract}
Objective: to investigate the factors related to infant deaths due to congenital anomalies in the municipalities belonging to the 14th Regional Health Region of Paraná, in the period from 2010 to 2019. Methodology: crosssectional study based on secondary data from the Born Information System Living, Mortality Information System and Notifiable Diseases Information System. Results: the detection of anomalies before hospital discharge proved to be a protective factor against death. The following factors were associated with death: children with low birth weight $(\mathrm{OR}=$ $5.7,95 \% \mathrm{CI}=3.37-9.77, \mathrm{p}<0.001)$ and children of mothers without a partner $(\mathrm{OR}=1.8,95 \% \mathrm{CI}=1.10-2.98, \mathrm{p}=0.019)$, inadequate number of prenatal consultations $(\mathrm{OR}=1.7,95 \% \mathrm{CI}=1.01-2.84, \mathrm{p}=0.047)$, double pregnancy and more $(\mathrm{OR}=9.3,95 \% \mathrm{CI}=1.63-53.52, \mathrm{p}=0.031)$, premature birth $(\mathrm{OR}=4.0,95 \% \mathrm{CI}=2.39-6.85, \mathrm{p}<0.001)$ and inadequate Apgar score within the first $(\mathrm{OR}=5.5,95 \% \mathrm{CI}=3.28-9.32, \mathrm{p}<0.001)$ and fifth minute of life $(\mathrm{OR}=8.9,95 \% \mathrm{CI}=4.46$ 17.59, $\mathrm{p}<0.001)$. Conclusion: several factors are associated with mortality from congenital anomalies. Therefore, the importance of preventing these factors through strengthening the process of epidemiological surveillance of
\end{abstract}


anomalies, family planning, adequate prenatal care and early diagnosis to improve the quality of life and survival of children with anomalies is emphasized.

Keywords: Congenital abnormalities; Risk factors; Infant mortality; Public health.

\begin{abstract}
Resumen
Objetivo: investigar los factores relacionados con las muertes infantiles por por anomalías congénitas en los municipios pertenecientes a el 14º Departamento Regional de Salud del estado de Paraná, en el período 2010 a 2019. Metodología: estudio transversal con base en datos secundarios del Sistema de Información de Nacidos Vivos, Sistema de Información sobre Mortalidad y Sistema de Información de Enfermedades Notificables. Resultados: la detección de anomalías antes del alta hospitalaria resultó ser un factor protector frente a la muerte. Los siguientes factores se asociaron con la muerte: niños con bajo peso al nacer $(O R=5,7$; IC95\%=3,37-9,77; p $<0,001)$ e hijos de madres sin pareja $(\mathrm{OR}=1,8 ; \mathrm{IC} 95 \%=1,10-2,98 ; \mathrm{p}=0,019)$, número inadecuado de consultas prenatales $(\mathrm{OR}=1,7$; IC95\%= 1,01-2,84; p=0,047), tipo de embarazo doble y más $(\mathrm{OR}=9,3 ; \mathrm{IC} 95 \%=1,63-53,52 ; \mathrm{p}=0,031)$, parto prematuro $(\mathrm{OR}=4,0 ; \mathrm{IC} 95 \%=2,39-6,85 ; \mathrm{p}<0,001)$ y puntaje de Apgar inadecuado en el primer $(\mathrm{OR}=5,5 ; \mathrm{IC} 95 \%=$ $3,28-9,32 ; \mathrm{p}<0,001)$ y quinto minuto de vida (OR = 8,9; IC del 95\% = 4,46-17,59; p <0,001). Conclusión: varios factores están asociados con la mortalidad por anomalías congénitas. Por tanto, se enfatiza la importancia de prevenir estos factores a través de fortalecimiento del proceso de vigilancia epidemiológica de anomalías, planificación familiar, atención prenatal adecuada y diagnóstico temprano para mejorar la calidad de vida y supervivencia de los niños con anomalías.
\end{abstract}

Palabras clave: Anomalías Congénitas; Factores de riesgo; Mortalidad infantil; Salud pública.

\title{
1. Introdução
}

Durante o desenvolvimento embrionário do ser humano, diferentes eventos anormais podem ocorrer como as mutações gênicas e cromossomopatias, resultando em Anomalias Congênitas no embrião, conhecidas também como Defeitos Congênitos (Mendes et al., 2018).

As anomalias congênitas são defeitos morfológicos e/ou funcionais de um órgão, parte de um órgão ou região maior do corpo os quais podem apresentar-se isolados, com a presença de apenas um defeito, ou associados, com a presença de dois ou mais defeitos. As anomalias quando denominadas menores podem ser frequentes na população geral e não acarretar problemas graves aos portadores. Porém, algumas trazem consequências médicas ou estéticas graves sendo denominadas de maiores (Mendes et al., 2018).

O risco de ocorrência dessas alterações pode aumentar com o número de casos ocorridos em determinada família e proximidade de parentesco com o afetado. Além do fator hereditário, fatores ambientais, comportamentais, biológicos, sociais e características maternas também podem ser associados à ocorrência de anomalias congênitas, sendo denominados de agentes teratogênicos. Um agente teratogênico é definido como qualquer substância, organismo, agente físico ou estado de deficiência que é capaz de alterar e afetar o desenvolvimento embrionário e fetal (Baldacci et al., 2018; Mendes et al., 2018).

Classificam-se como teratógenas causas externas como: forma de alimentação, uso de drogas lícitas e ilícitas, obesidade, ambientes frequentados, alguns medicamentos, algumas infecções, automedicação indevida como a utilização indiscriminada de anti-inflamatórios, a qualidade da assistência prestada à gestante, realização do pré-natal de forma adequada, tentativas de aborto e envolvimento com acidentes (Baldacci et al., 2018; Persson et al., 2017).

De acordo com a Organização Mundial da Saúde, a cada ano, aproximadamente 3,2 milhões de crianças em todo o mundo nascem com anomalias congênitas, destes recém-nascidos aproximadamente 300.000 morrem nos primeiros 28 dias de vida (WHO, 2016). Um estudo recente mostrou que as anomalias foram responsáveis por mais óbitos de crianças do que a malária em 2015, aproximadamente 0,5 milhões de óbitos (Wang et al., 2016).

Em países desenvolvidos como nos Estados Unidos, os defeitos congênitos figuram entre as principais causas de mortalidade infantil, representando 20,6\% das mortes infantis em 2017 (Ely \& Driscoll, 2019). Na Europa, as anomalias congênitas são a principal causa de mortalidade perinatal: a rede de Vigilância Europeia de Anomalias Congênitas estimou uma mortalidade perinatal associada as anomalias de 9,2 por 10.000 nascimentos no período de 2008 a 2012 (EUROCAT, 
2021).

Na Colômbia, foi identificado que as anomalias congênitas contribuem em grande proporção para a mortalidade neonatal todos os anos com uma taxa de mortalidade de 20,8 por 10.000 nascidos vivos no período de 1999-2008 (Roncancio et al., 2018). No Brasil, outro país sul americano e emergente, as anomalias congênitas figuraram entre as principais causas de óbito infantil, correspondendo à principal causa de morte nos estados das regiões Sul, Centro-Oeste e Sudeste, com exceção dos estados Minas Gerais e Goiás (França et al., 2017).

Diante do exposto, observa-se que a anomalias congênitas exercem grande influência sobre as estatísticas vitais infantis, no entanto há uma escassez de estudos sobre como as mesmas impactam na mortalidade infantil no Brasil. Logo, é perceptível a necessidade de investigação e posterior identificação dos fatores associados aos óbitos infantis por essa causa. Além disso, vários estudos questionam a necessidade de mais pesquisas sobre a temática e o rastreamento contínuo das anomalias a fim de reforçar a vigilância epidemiológica das mesmas para planejamento de ações preventivas conforme as fragilidades diagnosticadas por meio desse processo (Almli et al., 2020; Cavero-Carbonell et al., 2017; Lima et al., 2018).

Nesse contexto, a prevenção de mortes infantis por anomalias congênitas é uma maneira muito importante de contribuir para a redução da mortalidade neonatal e de menores de 5 anos, que são metas incluídas nos Objetivos de Desenvolvimento Sustentável (ODS) para 2030 na parte de saúde e bem-estar da Organização das Nações Unidas (UN, 2015).

Tendo em vista a relevância das anomalias congênitas para a saúde materno-infantil e concomitantemente a escassez de estudos epidemiológicos sobre a temática no Brasil o objetivo deste estudo foi investigar os fatores associados aos óbitos infantis por anomalias congênitas nos municípios pertencentes a $14^{\mathrm{a}}$ Regional de Saúde do estado do Paraná, Brasil, no período de 2010 a 2019.

\section{Metodologia}

Trata-se de um estudo transversal, com abordagem quantitativa (Estrela, 2018) baseado em dados secundários obtidos do Sistema de Informação dos Nascidos Vivos (Sinasc), do Sistema de Informação de Mortalidade (SIM) e do Sistema de Informação de Agravos de Notificação (Sinan) fornecidos pelo Departamento de Vigilância Epidemiológica da $14^{\mathrm{a}}$ Regional de Saúde de Paranavaí-PR. A população foi constituída por todas crianças portadoras de anomalias congênitas, residentes nos municípios da área de abrangência da 14ª Regional de Saúde que nasceram e morreram no período de 2010 a 2019.

A partir de 1999, o Ministério da Saúde introduziu um campo específico (campo 34) para o registro das anomalias congênitas na Declaração de Nascidos Vivos e seu código corresponde ao Capítulo XVII da Classificação Internacional das Doenças (CID-10) (OMS, 1997).

Com relação às variáveis, a mortalidade por anomalias congênitas foi definida como variável dependente, as variáveis independentes foram: sexo da criança, peso ao nascer, detecção de anomalia congênita ao nascer, estado conjugal da mãe, idade da mãe, escolaridade da mãe, ocupação da mãe, histórico de filho morto, número de consultas de pré-natal, tipo de gravidez, duração da gestação, tipo de parto, escore de Apgar no primeiro e no quinto minuto.

A variável "estado conjugal da mãe" foi dicotomizada como sem e com companheiro. "Com companheiro" incluíramse as mulheres propriamente ditas casadas, com união consensual e união estável e para "sem companheiro" incluíram-se as solteiras, separadas e divorciadas. Quanto à escolaridade materna considerou-se menos de oito anos de estudos versus oito ou mais anos de estudo (Magalhães et al., 2006).

O tempo de parto foi categorizado por meio da duração da gestação em prematuro ( $<37$ semanas) e a termo $(\geq 37$ semanas). O tipo de parto como normal ou cesárea. O número de consultas de pré-natal em inadequado (menos de sete consultas) e adequado (sete ou mais consultas). O sexo da criança como masculino e feminino. Os índices de Apgar no 
primeiro e no quinto minuto em inadequado (menor que sete) e adequado (igual ou maior que sete).

A raça/cor da criança foi categorizada dicotomicamente como branca e não branca sendo que para a não branca incluiu-se preta, amarela, parda e indígena. $\mathrm{O}$ peso ao nascer foi classificado em adequado, quando igual ou superior a 2.500 $\mathrm{kg}$, e em baixo peso, quando inferior.

Os tipos de anomalias congênitas que figuraram como causa básica dos óbitos infantis foram coletados por meio do SIM e agrupados nos seguintes grupos, de acordo com a CID 10: sistema neurológico (CID10 Q00.0, Q01.9, Q03.9, Q04.2, Q04.9 e Q05.9), sistema cardiovascular (CID10 Q20.3, Q20.4, Q20.8, Q20.9, Q21.1, Q21.3, Q22.0, Q22.5, Q22.6, Q23.4, Q24.0, Q24.6, Q24.8, Q24.9 e Q28.0), sistema musculoesquelético (CID10 Q32.0, Q75.0 e Q78.0), sistema urogenital (CID10 Q63.9), sistema pulmonar (CID10 Q33.6), aparelho digestivo e orofacial (CID10 Q37.9, Q39.0, Q39.1, Q40.0, Q41.8, Q41.9, Q43.8, Q43.9, Q45.9, Q60.2, Q60.6, Q61.3, Q62.0, Q79.0, Q79.2, Q79.3, Q79.4 e Q79.5) síndromes (CID 10 Q90.9, Q91.3, Q91.7 e Q99.9) e anomalias múltiplas (CID10 Q87.0, Q87.9, Q89.7, Q89.8 e Q89.9).

Os dados foram tabulados e codificados por meio do software Microsoft Excel 2016. Para verificar associação entre as variáveis e para as análises multivariadas empregou-se como estimativa o Odds Ratio (OR), adotando-se um nível de significância em 5\% ( $<<0,05)$, intervalos de confiança 95\%. Para análise estatística utilizou-se o software Statistica versão 10. Os resultados foram apresentados por meio de tabelas.

Para a realização do estudo foi solicitada autorização formal à Secretaria Estadual de Saúde do Estado do Paraná por meio da $14^{\text {a }}$ Regional de Saúde de Paranavaí para acesso aos dados secundários. O projeto de pesquisa foi aprovado pelo Comitê de Ética em Pesquisa Envolvendo Seres Humanos da Universidade Estadual de Maringá (UEM) (Parecer $n^{\circ}$ 3.211.733/20 de março de 2019) e pelo Comitê de Ética em Pesquisa do Hospital do Trabalhador da Secretaria de Estado da Saúde do Paraná (SESA-PR) (Parecer nº 3.440.478/05 de julho de 2019).

\section{Resultados}

O total das variáveis não informadas foram as seguintes: Um caso das variáveis "Sexo da criança", "Idade da mãe" e "Tipo de gravidez"; dois casos das variáveis "Escolaridade da mãe", "Ocupação da mãe" e "Tipo de parto"; quatro casos das variáveis "Peso ao nascer", "Detecção de anomalia congênita ao nascer", "Estado conjugal da mãe", "Número de consultas pré-natal" e "Apgar $1^{\circ}$ minuto"; 5 casos da variável "50 minuto"; e sete casos das variáveis "Histórico de filho morto" e "Tempo do parto". Totalizando 48 (17,02\%) casos não informados sendo que "Histórico de filho morto" e "Tempo do parto" obtiveram mais subnotificação.

Constatou-se que no período de 2010 a 2019 ocorreram 282 nascimentos com anomalias congênitas, sendo que destes, 125 evoluíram a óbito neste mesmo período, dentre os 28 municípios de residência da região da $14^{\mathrm{a}}$ Regional de Saúde de Paranavaí-PR. Todas as variáveis que apresentaram indicação "ignorado" ou que não apresentaram o campo preenchido foram consideradas como não informadas.

Dos nascimentos, $161(57,30 \%)$ apresentaram predomínio do sexo masculino, peso adequado $190(68,35 \%)$ e detecção de anomalia congênita ao nascer $220(79,14 \%)$. A maioria $182(65,47 \%)$ das mães tinha companheiro, idade $\geq 20$ anos 230 (81,85\%), $\geq 8$ anos de estudo 200 (71,68\%), com ocupação 147 (52,50) e sem histórico de filho morto $231(84 \%)$ (Tabela 1). 
Tabela 1 - Fatores maternos e infantis para a mortalidade dos casos de anomalia congênita nos municípios da $14^{\mathrm{a}}$ Regional de saúde de Paranavaí-PR, Brasil, 2010-2019.

\begin{tabular}{|c|c|c|c|c|c|}
\hline Variáveis & $\begin{array}{c}\text { TOTAL } \\
\text { N }(\%)\end{array}$ & $\begin{array}{l}\text { Óbito } \\
\text { N (\%) }\end{array}$ & $\begin{array}{c}\text { Não óbito } \\
\text { N (\%) }\end{array}$ & OR (IC95\%) & Valor de $p$ \\
\hline \multicolumn{6}{|l|}{ Sexo da criança } \\
\hline Feminino & $120(42,70)$ & $62(22,06)$ & $58(20,64)$ & Referência & \\
\hline Masculino & $161(57,30)$ & $62(22,06)$ & $99(35,23)$ & $0,6(0,36-0,94)$ & 0,028 \\
\hline Total & $281(100)$ & $124(44,13)$ & $157(55,87)$ & & \\
\hline \multicolumn{6}{|l|}{ Peso ao nascer } \\
\hline Peso adequado & $190(68,35)$ & $58(20,86)$ & $132(47,48)$ & Referência & \\
\hline Baixo Peso & $88(31,65)$ & $63(22,66)$ & $25(8,99)$ & $5,7(3,37-9,77)$ & $<0,001$ \\
\hline Total & $278(100)$ & $121(43,53)$ & $157(56,47)$ & & \\
\hline \multicolumn{6}{|c|}{ Detecção de AC ao nascer } \\
\hline $\operatorname{Sim}$ & $220(79,14)$ & $63(22,66)$ & $157(56,47)$ & Referência & \\
\hline Não & $58(20,86)$ & $58(20,86)$ & $0(00,00)$ & - & $<0,001$ \\
\hline Total & $278(100)$ & $121(43,53)$ & $157(56,47)$ & & \\
\hline \multicolumn{6}{|c|}{ Estado conjugal da mãe } \\
\hline Com companheiro & $182(65,47)$ & $70(25,18)$ & $112(40,29)$ & Referência & \\
\hline Sem companheiro & $96(34,53)$ & $51(18,35)$ & $45(16,19)$ & $1,8(1,10-2,98)$ & 0,019 \\
\hline Total & $278(100)$ & $121(43,53)$ & $157(56,47)$ & & \\
\hline \multicolumn{6}{|l|}{ Idade da mãe } \\
\hline$<20$ anos & $51(18,15)$ & $21(7,47)$ & $30(10,68)$ & Referência & \\
\hline$\geq 20$ anos & $230(81,85)$ & $103(36,65)$ & $127(45,20)$ & $1,2(0,63-2,14)$ & 0,639 \\
\hline Total & $281(100)$ & $124(44,13)$ & $157(55,87)$ & & \\
\hline \multicolumn{6}{|c|}{ Escolaridade da mãe } \\
\hline$\geq 8$ anos estudo & $200(71,68)$ & $92(32,97)$ & $108(38,71)$ & Referência & \\
\hline$<8$ anos estudo & $79(28,32)$ & $31(11,11)$ & $48(17,20)$ & $0,8(0,45-1,29)$ & 0,306 \\
\hline Total & $279(100)$ & $123(44,09)$ & $156(55,91)$ & & \\
\hline \multicolumn{6}{|l|}{ Ocupação da mãe } \\
\hline Sem ocupação & $133(47,50)$ & $58(20,71)$ & $75(26,79)$ & Referência & \\
\hline Com ocupação & $147(52,50)$ & $65(23,21)$ & $82(29,29)$ & $1,0(0,64-1,64)$ & 0,918 \\
\hline Total & $280(100)$ & $123(43,93)$ & $157(56,07)$ & & \\
\hline \multicolumn{6}{|c|}{ Histórico de filho morto } \\
\hline Sem histórico & $231(84,00)$ & $103(37,45)$ & $128(46,55)$ & Referência & \\
\hline Com histórico & $44(16,00)$ & $21(7,64)$ & $23(8,36)$ & $1,1(0,59-$ & 0,701 \\
\hline Total & $275(100)$ & $124(45,09)$ & $151(54,91)$ & $2,16)$ & \\
\hline
\end{tabular}

$\mathrm{OR}=$ Odds ratio; $\mathrm{IC}=$ Intervalo de confiança

$p=$ Nível descritivo para o Teste Qui-quadrado de Pearson $\left(\mathrm{X}^{2}\right)$

Fonte: SIM, Sinasc e Sinan, $14^{\text {a }}$ Regional de Saúde de Paranavaí-PR.

Ainda na Tabela 1, identificou-se que os fatores estatisticamente associados ao óbito foram seguintes: sexo da criança, peso ao nascer, detecção de anomalia congênita ao nascer e estado conjugal da mãe (Tabela 1).

Em relação à gestação e ao parto, de forma geral, as seguintes características foram predominantes: pré-natal adequado 197 (70,86\%), tipo de gravidez única 273 (97,15\%), parto cesáreo 237 (84,64\%), a termo 189 (68,73), e Apgar no $1^{\circ}$ e $5^{\circ}$ minuto adequados $184(66,19 \%)$ e $226(81,59 \%)$ respectivamente (Tabela 2$)$.

Pode-se observar que os seguintes fatores se associaram com o óbito: número de consultas pré-natal, tipo de gravidez, tempo de parto e Apgar no $1^{\circ}$ e $5^{\circ}$ minuto (Tabela 2). 
Tabela 2 - Fatores obstétricos e neonatais para a mortalidade dos casos de anomalia congênita nos municípios da $14^{\mathrm{a}}$ Regional de saúde de Paranavaí-PR, Brasil, 2010-2019

\begin{tabular}{|c|c|c|c|c|c|}
\hline Variáveis & $\begin{array}{c}\text { TOTAL } \\
\mathbf{N}(\%)\end{array}$ & $\begin{array}{l}\text { Óbito } \\
\text { N (\%) }\end{array}$ & $\begin{array}{c}\text { Não óbito } \\
\text { N (\%) }\end{array}$ & OR (IC95\%) & Valor de $p$ \\
\hline \multicolumn{6}{|c|}{ Número de consultas pré natal } \\
\hline Adequado & $197(70,86)$ & $79(28,42)$ & $118(42,45)$ & Referência & \\
\hline Inadequado & $81(29,14)$ & $43(15,47)$ & $38(13,67)$ & $1,7(1,01-2,84)$ & 0,047 \\
\hline Total & $278(100)$ & $122(43,88)$ & $156(56,12)$ & & \\
\hline \multicolumn{6}{|l|}{ Tipo de gravidez } \\
\hline Única & $273(97,15)$ & $117(41,64)$ & $156(55,52)$ & Referência & \\
\hline Dupla e mais & $8(2,85)$ & $7(2,49)$ & $1(0,36)$ & $9,3(1,63-53,52)$ & $0,031 *$ \\
\hline Total & $281(100)$ & $124(44,13)$ & $157(55,87)$ & & \\
\hline \multicolumn{6}{|l|}{ Tipo de parto } \\
\hline Vaginal & $43(15,36)$ & $15(5,36)$ & $28(10,00)$ & Referência & \\
\hline Cesáreo & $237(84,64)$ & $108(38,57)$ & $129(46,07)$ & $1,6(0,80-3,07)$ & 0,194 \\
\hline Total & $280(100)$ & $123(43,93)$ & $157(56,07)$ & & \\
\hline \multicolumn{6}{|l|}{ Tempo de parto } \\
\hline A termo & $189(68,73)$ & $64(23,27)$ & $125(45,45)$ & Referência & \\
\hline Prematuro & $86(31,27)$ & $58(21,09)$ & $28(10,18)$ & $4,0(2,39-6,85)$ & $<0,001$ \\
\hline Total & $275(100)$ & $122(44,36)$ & $153(55,64)$ & & \\
\hline \multicolumn{6}{|l|}{ Apgar $1^{\circ}$ minuto } \\
\hline Adequado & $184(66,19)$ & $55(19,78)$ & $129(46,40)$ & Referência & \\
\hline Inadequado & $94(33,81)$ & $66(23,74)$ & $28(10,07)$ & $5,5(3,28-9,32)$ & $<0,001$ \\
\hline Total & $278(100)$ & $121(43,53)$ & $157(56,47)$ & & \\
\hline \multicolumn{6}{|l|}{ Apgar $5^{\circ}$ minuto } \\
\hline Adequado & $226(81,59)$ & $78(28,16)$ & $148(53,43)$ & Referência & \\
\hline Inadequado & $51(18,41)$ & $42(15,16)$ & $9(3,25)$ & $8,9(4,46-17,59)$ & $<0,001$ \\
\hline Total & $277(100)$ & $120(43,32)$ & (157) 56,68 & & \\
\hline
\end{tabular}

$\mathrm{OR}=$ Odds ratio; $\mathrm{IC}=$ Intervalo de confiança

Teste Qui-quadrado de Pearson $\left(\mathrm{X}^{2}\right)$

* Nível descritivo para o Teste Exato de Fisher

Fonte: SIM, Sinasc e Sinan, $14^{\text {a }}$ Regional de Saúde de Paranavaí-PR

Das crianças que morreram a maioria apresentou idade média de 70,1 $( \pm 156,22)$ dias e os seguintes tipos de anomalias como causa básica de morte: cardiovasculares 43 (34,40), do aparelho digestivo e orofaciais 26 (20,80), neurológicas 20 (16,00), múltiplas $13(10,40)$, síndromes 13 (10,40). Destes 125 óbitos infantis por anomalias congênitas apenas 70 (56,00\%) foram registrados como investigados pelo comitê de mortalidade.

\section{Discussão}

As crianças com Baixo Peso (BP) ao nascer apresentaram 5,7 (IC95\% 3,37-9,77; p<0,001) maior chance de morrer quando comparadas aquelas com peso adequado. Resultado semelhante ao de um estudo realizado nos Estados Unidos em que foi identificado que recém-nascidos portadores de anomalias congênitas com baixo peso tiveram sobrevida significativamente reduzida e piores desfechos clínicos em comparação com os que nasceram com peso normal (Raymond et al., 2020).

O BP ao nascer é considerado um complexo problema de saúde pública por sua etiologia multifatorial e associação com fatores sociodemográficos, biológicos e comportamentais. Além disso, é um importante indicador de risco para mortalidade infantil e contribui para desenvolvimento de morbidades como doenças infecciosas, alterações cognitivas e 
neurológicas, atraso do desenvolvimento e maior probabilidade de desenvolver doenças crônicas (Marin et al., 2021; Tourinho \& Reis, 2012).

Além disso, as análises mostram que a detecção de anomalia congênita ao nascer, ou seja, no período perinatal, é um fator de proteção para o óbito. O que corrobora com outros estudos que tiveram a maioria dos casos de anomalias diagnosticados depois do período pré-natal (Zhang et al., 2020). Dessa forma, embora a anomalia tenha sido detectada percebese um déficit na detecção precoce da mesma, ou seja, no período pré-natal.

Estudos mostram que certos casos de anomalias congênitas são detectados por ultrassom no pré-natal e outros por meio do exame físico no recém-nascido, porém, nem todos os bebês são diagnosticados antes da alta hospitalar (Liberman et al., 2014; Peterson et al., 2014). Vale ressaltar que a detecção precoce das mesmas contribui para prevenção de agravos a saúde do recém-nascido com a doença, inclusive de óbitos. Dessa maneira, colabora para a redução da morbimortalidade infantil, principalmente neonatal precoce, assim como para o manejo da doença e o planejamento de assistência em saúde a fim de melhorar a qualidade de vida e a sobrevida das crianças nascidas com anomalias congênitas (Cosme et al., 2017).

Todavia, uma pesquisa ressaltou que nem sempre a realização do diagnóstico durante o período pré-natal pode contribuir para o aumento da sobrevida de crianças diagnosticadas com alguma AC, visto que muitas vezes não é dada uma sequência na assistência em saúde após a detecção da anomalia, por exemplo, em casos de necessidade de intervenção cirúrgica imediata (Özer Bekmez et al., 2019).

Dessa maneira, vale salientar que existem diversos fatores externos que influenciam no diagnóstico das anomalias como o ambiente, a demanda nos serviços de saúde e a falta de aparato tecnológico. Nesse contexto, um exemplo disso, é a atual pandemia do COVID-19 que gerou muitos impactos negativos para a vigilância epidemiológica das anomalias congênitas, inclusive para o diagnóstico precoce das mesmas (Ludorf et al., 2020).

Além disso, o momento de dar a notícia do diagnóstico de anomalia congênita é muito delicado para os pais e para a família, o qual pode desencadear excessiva ansiedade e prejuízos psicológicos, agravando-se com a demora. Apesar de todas essas questões envolvidas com a notícia do diagnóstico da doença, muitas vezes a mesma não é repassada para a mãe e para a família de forma adequada e humanizada, levando em consideração apenas a condição biológica da criança e não considerando os aspectos biopsicossociais da família (Fontoura et al., 2018; Oliveira et al., 2019).

Em relação às mães, os resultados evidenciaram que aquelas sem companheiro, apresentaram maior chance de que seus filhos com anomalias congênitas morressem, quando se comparadas às "com companheiro" (Tabela 1). No presente estudo a maioria das gestantes tinham companheiro, assim como observado em um estudo realizado no Rio Grande do Sul, onde foi identificada uma tendência de ascendência das anomalias congênitas para mulheres casadas ou em união consensual. Assim como em outro estudo realizado na Paraíba onde a maioria das gestantes com filhos com anomalias estava em uma união consensual (Luz et al., 2019).

Uma pesquisa realizada na África demonstrou que gestantes que recebem apoio dos parceiros durante a gestação tem maior probabilidade de utilizar as recomendações do pré-natal. Nesse contexto, ainda segundo o estudo, o apoio do parceiro mostrou ter um efeito significativo na adoção das medidas preventivas de anomalias congênitas no pré-natal como a utilização do ácido fólico (Asresu et al., 2019).

Nessa lógica, sugere-se que gestantes sem companheiros apresentam maior chance de terem recém-nascido com complicações, enquanto que as que contam com a presença e o suporte dos mesmos possuem fator de proteção contra essas situações (Asresu et al., 2019). Todavia, evidencia-se que a minoria das mães possui esse suporte do parceiro (Asresu et al., 2019; Surkan et al., 2019).

No que tange as características da gestação e do parto, as mulheres que tiveram número de consultas de pré-natal inadequado apresentaram maior chance de que seus filhos com anomalias congênitas morressem. Pesquisas concordam que os 
diagnósticos precoces realizados durante a assistência pré-natal, assim como as consultas multiprofissionais, contribuem para a redução da mortalidade por anomalias congênitas críticas (Combs et al., 2020; Qiu et al., 2020).

Em relação ao tipo de gravidez, o presente estudo apresenta que a gravidez múltipla proporciona maior chance de mortalidade por anomalias congênitas. O que vai ao encontro de uma pesquisa realizada na cidade de Maringá, Paraná, Brasil, que identificou como fatores de risco para a mortalidade neonatal, entre mulheres com gestação de alto risco, a gestação múltipla na gestação atual (Qiu et al., 2020).

As crianças com anomalias congênitas de parto prematuro apresentaram maior chance de morrer, o que concorda com um estudo realizado nos Estados Unidos em que a maioria dos recém-nascidos com anomalias congênitas que nasceram prematuros morreram (Ely \& Driscoll, 2019).

No que tange ao Apgar presente estudo apresenta que crianças com anomalias congênitas que tiveram Apgar no $1^{\circ} \mathrm{e}$ $5^{\circ}$ minutos inadequados têm maior chance de morrer. Corroborando com um estudo em que foi realizado análises do modelo de regressão logística que revelaram que maior índice de Apgar em 5 minutos foi associado a risco reduzido de mortalidade para recém-nascidos com anomalias congênitas (Magalhães et al., 2006).

\section{Considerações Finais}

Dessa forma, por meio da presente pesquisa pode-se concluir que fatores, além da hereditariedade e genética, estão relacionados com a mortalidade por anomalias congênitas como sexo da criança, peso ao nascer, detecção de anomalias congênitas ao nascer, estado conjugal da mãe, número de consultas pré-natal, tipo de gravidez, tempo de parto e Apgar no $1^{\circ} \mathrm{e}$ $5^{\circ}$ minuto.

Dessa maneira, observa-se a importância do conhecimento dessas informações pelos profissionais de saúde a fim de que possam atuar na prevenção das anomalias congênitas durante o planejamento familiar e período pré-natal, na deteç̧ão precoce das mesmas e na elaboração de plano de cuidados a fim de melhorar a qualidade de vida, reduzir a mortalidade e aumentar a taxa de sobrevida da criança portadora de anomalia.

Durante o estudo foram observadas lacunas no preenchimento da notificação do nascimento da criança com anomalia congênita e também da declaração de óbito, o que dificulta o processo de vigilância epidemiológica para as anomalias. Também pode-se observar a demora e a falta de investigação de óbitos infantis. Ambos foram fatores de limitação para o presente estudo. Logo, ressalta-se a relevância e necessidade de um preenchimento adequado das notificações de nascimento e de óbito pelos profissionais de saúde a fim de assegurar uma vigilância eficiente das anomalias.

Além disso, fazem-se necessários mais estudos sobre o tema, para que subsidiem a implantação de um processo de rastreamento eficiente das anomalias congênitas durante o planejamento familiar e pré-natal assim como, políticas públicas que movimentem serviços de saúde e profissionais de saúde para a prevenção das anomalias congênitas durante todo o período gestacional e a promoção de qualidade de vida para as crianças com anomalias congênitas dentro das redes de atenção à saúde do Sistema Único de Saúde.

\section{Referências}

Almli, L. M., Ely, D. M., Ailes, E. C., Abouk, R., Grosse, S. D., Isenburg, J. L., Waldron, D. B., \& Reefhuis, J. (2020). Infant Mortality Attributable to Birth Defects - United States, 2003-2017. MMWR. Morbidity and mortality weekly report, 69(2), 25-29. http://dx.doi.org/10.15585/mmwr.mm6902a1.

Asresu, T. T., Hailu, D., Girmay, B., Abrha, M. W., \& Weldearegay, H. G. (2019). Mothers' utilization and associated factors in preconception care in northern Ethiopia: A community based cross sectional study. BMC pregnancy and childbirth, 19(1), 1-7. https://doi.org/10.1186/s12884-019-2478-1.

Baldacci, S., Gorini, F., Santoro, M., Pierini, A., Minichilli, F., \& Bianchi, F. (2018). Environmental and individual exposure and the risk of congenital anomalies: a review of recent epidemiological evidence. Epidemiologia e prevenzione, 42(3-4 Suppl 1), 1-34. http://www.epiprev.it/pubblicazione/epidemiolprev-2018-42-3-4-suppl-1. 
Cavero-Carbonell, C., Vinkel-Hansen, A., Rabanque-Hernández, M. J., Martos, C., \& Garne, E. (2017). Fetal exposure to montelukast and congenital anomalies: a population based study in Denmark. Birth defects research, 109(6), 452-459. https://doi.org/10.1002/bdra.23621.

Combs, C. A., Hameed, A. B., Friedman, A. M., Hoskins, I. A., Quality Committee, \& Society for Maternal-Fetal Medicine. (2020). Special statement: Proposed quality metrics to assess accuracy of prenatal detection of congenital heart defects. American journal of obstetrics and gynecology, 222(6), B2-B9. https://doi.org/10.1016/j.ajog.2020.02.040.

Cosme, H. W., Lima, L. S., \& Barbosa, L. G. (2017). Prevalência de anomalias congênitas e fatores associados em recém-nascidos do município de São Paulo no período de 2010 a 2014. Revista Paulista de Pediatria, 35, 33-38. https://doi.org/10.1590/1984-0462/;2017;35;1;00002.

Ely, D. M., \& Driscoll, A. K. (2019). Infant Mortality in the United States, 2017: Data From the Period Linked Birth/Infant Death File. National vital statistics reports: from the Centers for Disease Control and Prevention, National Center for Health Statistics, National Vital Statistics System, 68(10), 1-20. https://stacks.cdc.gov/view/cdc/80304.

Estrela, C. (2018). Metodologia Científica: Ciência, Ensino, Pesquisa. (3a ed.), Artes Médicas.

European Surveillance of Congenital Anomalies-EUROCAT. (2021). Key Public Health Indicators tables. https://eu-rdplatform.jrc.ec.europa.eu/eurocat/eurocat-data/key-public-health-indicators_en.

Fontoura, F. C., Cardoso, M. V. L. M. L., Rodrigues, S. E., Almeida, P. C. D., \& Carvalho, L. B. (2018). Anxiety of mothers of newborns with congenital malformations in the pre-and postnatal periods. Revista latino-americana de enfermagem, 26, e3080. https://doi.org/10.1590/1518-8345.2482.3080.

França, E. B., Lansky, S., Rego, M. A. S., Malta, D. C., França, J. S., Teixeira, R., \& Vasconcelos, A. M. N. (2017). Leading causes of child mortality in Brazil, in 1990 and 2015: estimates from the Global Burden of Disease study. Revista brasileira de Epidemiologia, 20, 46-60. https://doi.org/10.1590/19805497201700050005 .

Liberman, R. F., Getz, K. D., Lin, A. E., Higgins, C. A., Sekhavat, S., Markenson, G. R., \& Anderka, M. (2014). Delayed diagnosis of critical congenital heart defects: trends and associated factors. Pediatrics, 134(2), e373-e381. https://doi.org/10.1542/peds.2013-3949.

Lima, N. A., Silva, C. D. F., Santos, M. M. D., Fernandes, T. A. A. D. M., \& Lucena, E. E. D. S. (2018). Perfil Epidemiológico das Malformações Congênitas em Recém-Nascidos no Estado do Rio Grande do Norte no Período de 2004 a $2011 . \quad$ Rev. bras. ciênc. saúde, 45-50. https://periodicos.ufpb.br/index.php/rbcs/article/view/27529/19489.

Ludorf, K. L., Salemi, J. L., Kirby, R. S., Tanner, J. P., \& Agopian, A. J. (2020). Perspectives on challenges and opportunities for birth defects surveillance programs during and after the COVID-19 era. Birth defects research, 112(14), 1039-1042. https://doi.org/10.1002/bdr2.1710.

Luz, G. D. S., Karam, S. D. M., \& Dumith, S. C. (2019). Anomalias congênitas no estado do Rio Grande do Sul: análise de série temporal. Revista Brasileira de Epidemiologia, 22, e190040. http://dx.doi.org/10.1590/1980-549720190040.

Magalhães, M. D. L. C., Furtado, F. M., Nogueira, M. B., Carvalho, F. H. C., Almeida, F. M. L. D., Mattar, R., \& Camano, L. (2006). Gestação na adolescência precoce e tardia: há diferença nos riscos obstétricos?. Revista Brasileira de Ginecologia e Obstetrícia, 28, 446-452. http://dx.doi.org/10.1590/S0100-72032006000800002.

Marin, L. F. M., Antunes, F. T. T., Grivicich, I., \& Camassola, M. (2021). Malformações congênitas em nascidos vivos e fatores de risco materno-fetal em uma maternidade referência do estado do Tocantins, Brasil. Research, Society and Development, 10(7), e31310716679-e31310716679. https://doi.org/10.33448/rsd-v10i7.16679.

Mendes, I. C., Jesuino, R. S. A., Pinheiro, D. D. S., \& Rebelo, A. C. S. (2018). Anomalias congênitas e suas principais causas evitáveis: uma revisão. Revista Médica de Minas Gerais, 28, 1-6. http://dx.doi.org/10.5935/2238-3182.20180011.

Oliveira, P. S. D., Lamy, Z. C., Guimarães, C. N. M., Rodrigues, C. B., Silva, A. A. M. D., Simões, V. M. F., \& Sousa, P. D. S. (2019). Experiências de pais de crianças nascidas com microcefalia, no contexto da epidemia de Zika, a partir da comunicação do diagnóstico. Cadernos de Saúde Pública, 35 , e00226618. https://doi.org/10.1590/0102-311x00226618.

Organização Mundial da Saúde-OMS. (1997). Classificação Estatística Internacional de Doenças e Problemas Relacionados à Saúde, Décima Revisão (CID 10). São Paulo: Editora da Universidade de São Paulo.

Özer Bekmez, B., Alyamaç Dizdar, E., Okur, N., Büyüktiryaki, M., Uraş, N., \& Oğuz, S. S. (2019). Does prenatal diagnosis of critical congenital heart diseases influence the prereferral mortality in a center without surgical intervention?. The Journal of Maternal-Fetal \& Neonatal Medicine, 32(20), 3431-3434. https://doi.org/10.1080/14767058.2018.1465551.

Persson, M., Cnattingius, S., Villamor, E., Söderling, J., Pasternak, B., Stephansson, O., \& Neovius, M. (2017). Risk of major congenital malformations in relation to maternal overweight and obesity severity: cohort study of 1.2 million singletons. bmj, 357. https://doi.org/10.1136/bmj.j2563.

Peterson, C., Ailes, E., Riehle-Colarusso, T., Oster, M. E., Olney, R. S., Cassell, C. H., \& Gilboa, S. M. (2014). Late detection of critical congenital heart disease among US infants: estimation of the potential impact of proposed universal screening using pulse oximetry. JAMA pediatrics, 168(4), 361-370. https://doi.org/10.1001/jamapediatrics.2013.4779.

Qiu, X., Weng, Z., Liu, M., Chen, X., Wu, Q., Ling, W., \& Lin, Y. (2020). Prenatal diagnosis and pregnancy outcomes of 1492 fetuses with congenital heart disease: role of multidisciplinary-joint consultation in prenatal diagnosis. Scientific reports, 10(1), 1-11. https://doi.org/10.1038/s41598-020-64591-3.

Raymond, S. L., Hawkins, R. B., Peter, S. D. S., Downard, C. D., Qureshi, F. G., Renaud, E., \& Islam, S. (2020). Predicting morbidity and mortality in neonates born with gastroschisis. Journal of Surgical Research, 245, 217-224. https://doi.org/10.1016/j.jss.2019.07.065. 
Research, Society and Development, v. 10, n. 10, e58101018421, 2021

(CC BY 4.0) | ISSN 2525-3409 | DOI: http://dx.doi.org/10.33448/rsd-v10i10.18421

Roncancio, C. P., Misnaza, S. P., Peña, I. C., Prieto, F. E., Cannon, M. J., \& Valencia, D. (2018). Trends and characteristics of fetal and neonatal mortality due to congenital anomalies, Colombia 1999-2008. The Journal of Maternal-Fetal \& Neonatal Medicine, 31(13), 1748-1755. https://doi.org/10.1080/14767058.2017.1326901.

Surkan, P. J., Dong, L., Ji, Y., Hong, X., Ji, H., Kimmel, M., \& Wang, X. (2019). Paternal involvement and support and risk of preterm birth: findings from the Boston birth cohort. Journal of Psychosomatic Obstetrics \& Gynecology, 40(1), 48-56. https://doi.org/10.1080/0167482X.2017.1398725.

United Nations-UN. (2015). Sustainable Development Goals. https://sdgs.un.org/2030agenda.

Tourinho, A. B., \& Reis, M. L. B. D. S. (2012). Peso ao nascer: uma abordagem nutricional. Comun. ciênc. saúde, 19-30. https://bvsms.saude.gov.br/bvs/periodicos/revista_ESCS_v23_n1_a02_peso_ao_nascer.pdf.

Wang, H., Naghavi, M., Allen, C., Barber, R. M., Bhutta, Z. A., Carter, A., \& Bell, M. L. (2016). Global, regional, and national life expectancy, all-cause mortality, and cause-specific mortality for 249 causes of death, 1980-2015: a systematic analysis for the Global Burden of Disease Study 2015. The lancet, 388(10053), 1459-1544. https://doi.org/10.1016/S0140-6736(16)31012-1.

World Health Organization-WHO. (2016). Congenital anomalies fact sheet. http://www.who.int/news-room/fact-sheets/detail/congenital-anomalies.

Zhang, X., Chen, L., Wang, X., Wang, X., Jia, M., Ni, S., \& Zhu, S. (2020). Changes in maternal age and prevalence of congenital anomalies during the enactment of China's universal two-child policy (2013-2017) in Zhejiang Province, China: An observational study. PLoS medicine, 17(2), e1003047. https://doi.org/10.1371/journal.pmed.1003047. 